\title{
Sleep apnoea management in Europe during the COVID-19 pandemic: data from the European Sleep Apnoea Database (ESADA)
}

To the Editor:

Sleep disordered breathing (SDB) is highly prevalent, with a male to female predominance of two to one, and is more common in middle-aged and elderly subjects [1]. Affected patients often present with comorbidities such as obesity, cardiovascular disease (systemic hypertension, heart failure, atrial fibrillation) and diabetes mellitus type II [2]. The strong overlap between the profile for SDB patients and the identified risk factors for adverse outcomes of coronavirus disease 2019 (COVID-19) infection, which include age, male sex and cardiometabolic comorbidity [3], suggest that SDB patients may benefit from effective therapy if confronted with COVID-19 infection [4].

The COVID-19 pandemic has fundamentally changed the operation of healthcare systems around the globe. Resources have understandably been shifted from elective care to acute management of severely ill patients with life-threatening COVID-19 infection [5]. According to current recommendations, sleep medicine services are advised to reduce in-house services, and to provide medical care by remote contact using phone, video calls and telemedicine solutions [6-9].

In the current study, we assessed the impact of the COVID-19 pandemic on the management of SDB patients in Europe. We approached the centres of the European Sleep Apnoea Database (ESADA) cohort, a well-established network of sleep centres in 19 European countries [2], and a subsample of accredited sleep centres of the German Sleep Society (DGSM). The aim was to analyse how recommendations from expert organisations were applied across various European regions, and whether specific mitigation strategies were already in practice.

A purpose-built questionnaire addressed information about changes in clinical routines imposed during the COVID-19 pandemic compared with routine practices. Specifically, details on the diagnosis of SDB, the procedures for titration of positive airway pressure (PAP) treatment, and the follow-up of PAP treatment were addressed. One question sought the estimated proportion of staff still active in the sleep service during the pandemic compared with beforehand (0-100\%, for both "physicians" and "nurses/ technicians"). Finally, access to regional or national COVID-19-related guidelines for patients with SDB and for caregivers in sleep medicine services was evaluated. Actual numbers of confirmed COVID-19-infected inhabitants and reported COVID-19 deaths were computed per million inhabitants for each country based on information provided by the Johns Hopkins University COVID-19 statistics website (https://coronavirus.jhu.edu/map.html; 12 April 2020).

Data were provided by 25 of 29 ESADA centres and 15 of 283 DGSM-accredited sleep centres (table 1). Most centres were linked to a pulmonary department and all bar one were hospital based. In 31 of the total 40 participating centres, patients were unable to physically attend because of sleep centre or travel restrictions.

@ERSpublications

This study identified an $80 \%$ shutdown of sleep apnoea management throughout Europe. Most services have been limited to phone-based follow-up and the management of high-priority cases. Mitigation strategies appear to be insufficiently exploited. https://bit.ly/2KWf3IY

Cite this article as: Grote L, McNicholas WT, Hedner J. Sleep apnoea management in Europe during the COVID-19 pandemic: data from the European Sleep Apnoea Database (ESADA). Eur Respir J 2020; 55: 2001323 [https://doi.org/10.1183/13993003.01323-2020]. 
TABLE 1 Characteristics of the participating sleep centres

\begin{tabular}{lc} 
Number of centres & 40 \\
Total & 25 \\
European Sleep Apnoea Database & 15 \\
German Sleep Society & 5 \\
Distribution across Europe & 15 \\
$\quad$ Regions & 3 \\
Countries & 2 \\
Region of centres & 22 \\
$\quad$ North & 10 \\
West & 3 \\
Central & 22 \\
South & 18 \\
East & 32 \\
Clinical affiliation of centres & 32 \\
Other department\# & 8 \\
Patient spectrum of centres & \\
Entire spectrum of sleep/wake disorders & \\
Focus on sleep disordered breathing & \\
\hline \# : neurology (n=1); ear, nose and throat (n=3); cardiology (n=1); independent unit at hospital (n=12); \\
independent unit outside a hospital (n=1).
\end{tabular}

\section{TABLE 2 Change of activities during the COVID-19 pandemic compared with prior to the} pandemic

Prior

During

\begin{tabular}{lcc}
\hline Sleep apnoea diagnostic procedures & & \\
Polysomnography in lab & 92.5 & 20.0 \\
Polygraphy at home & 87.5 & 32.5 \\
Telemedicine-based & 30.0 & 27.5 \\
CPAP treatment start procedures & & 17.5 \\
In-lab titration & 90.0 & $22.5^{\#}$ \\
Ambulatory titration & 55.0 & 32.5 \\
Telemedicine-based APAP titration & 32.5 & 4 \\
$\quad$ Regularly use telemedicine $\mathrm{n}$ & 6 & 17.5 \\
Bi-level PAP treatment start procedures & & 17.5 \\
In-lab titration & 87.5 & 12.5 \\
Ambulatory titration & 40.0 & 3 \\
Telemedicine-based titration & 20.0 & \\
Regularly use telemedicine $\mathrm{n}$ & 4 & 7.5 \\
Follow-up routines for PAP treatment & & $17.5^{\#}$ \\
In-lab follow-up & 82.5 & 75.0 \\
Ambulatory titration & 92.5 & 57.5 \\
Distance follow-up & & 12 \\
$\quad$ Phone calls & 70 & 8 \\
$\quad$ Telemonitoring & 50.0 & 4 \\
Regularly use telemonitoring $\mathrm{n}$ & 7 &
\end{tabular}

Data are presented as \%, unless otherwise stated. All questions asked whether activities were performed regularly or rarely. CPAP: continuous positive airway pressure; APAP: automatic positive airway pressure; PAP: positive airway pressure. " : all rarely.

Diagnostic routines were changed substantially by the pandemic. Prior to the pandemic, laboratory-based polysomnography was performed "rarely or regularly" in $92.5 \%$ of centres, but this was only performed in $20 \%$ of centres during the pandemic $(\mathrm{p}<0.001$; table 2$)$. Conversely, telemedicine-based sleep apnoea diagnosis was used in $30.0 \%$ of centres prior to the pandemic and this number was only marginally reduced to $27.5 \%$ during the pandemic. From the 10 centres regularly using telemedicine-based diagnosis, 
three stopped the service during the pandemic, four maintained the level of use, and three increased use from rarely to regularly. Only two centres started this routine during the pandemic.

Prior to the pandemic, laboratory-based continuous positive airway pressure (CPAP) or bi-level PAP titration and initiation were practised in almost all centres but less than one fifth of centres continued this routine during the pandemic $(\mathrm{p}<0.001$; table 2$)$. In the 13 centres already practising telemedicine-based CPAP titration, four stopped the service, one reduced the use, six maintained the level of use, and two increased the use from rarely to regularly. Four centres started this routine during the pandemic.

Prior to the pandemic, 39 centres reported regular follow-up procedures in patients with SDB. This service continued in 36 centres during the pandemic but the mode of follow-up had changed (table 2). Specifically, three centres offered in-laboratory and seven centres performed ambulatory follow-up sleep recordings in selected patients. 30 centres provided follow-up by phone or video calls. Out of the nine centres without phone access, two practised telemedicine follow-up and four centres provided the support via home care providers. The remaining three centres offered no access to follow-up during the pandemic. Telemedicine-based follow-up was practised by a minority of centres.

Staffing levels in the sleep medicine service were reduced to $25 \%$ (interquartile range (IQR) $40 \%$ ) for physicians and to 19\% (IQR 28\%) for nurses/technicians, compared to pre-pandemic levels. Staffing reductions varied across European regions, being least in northern and most in the southern and central parts of Europe. However, there was no association identified between remaining staff and numbers of infected individuals or numbers of deaths relating to COVID-19 infection in each country. In addition, national recommendations or guidelines for patients with SDB were available in $45 \%$ of centres $(0 \%$ in eastern, $39 \%$ in central, $60 \%$ in southern and $100 \%$ in northern and western European centres). Only $28 \%$ of centres had guidelines for sleep service personnel.

This descriptive report highlights several important findings. First, sleep medicine services have been reduced by almost $80 \%$ during the first 1-2 months of the COVID-19 pandemic in Europe. Secondly, more comprehensive sleep studies using polysomnography or in-laboratory PAP titrations have been completely interrupted or practised only to a very limited extent in highly selected patient groups. Thirdly, commencement of treatment for SDB by various types of PAP therapy is equally reduced in the vast majority of centres and countries. Fourthly, patient follow-up is mainly managed by phone-based patient contacts. Fifthly, the full potential of mitigation strategies available by telemedicine has not been explored.

The sharp reduction in sleep medicine services in this study was expected and corresponds to the practice in other areas of medicine [3]. Prevention of virus spread at the sleep centres, such as by PAP-induced aerosol spread [10], as well as quarantine restrictions are plausible explanations for this decline. Nonetheless, we identified sizeable variations within and between countries in the lockdown of sleep medicine services. Rather unexpectedly, the use of telemedicine and other innovative technical solutions, including disposable diagnostic tools and non-contact sleep surveillance for sleep apnoea diagnosis, were not reported to any major extent. Uncertainty relating to data protection laws and inadequate practical experience are believed to limit dissemination of such new technology.

There is a medical need for the continued management of SDB patients. Most clinical case series regarding severely affected COVID-19 patients report clinical features including male predominance, obesity and cardiometabolic disorders [3], all of which are strongly associated with SDB. Furthermore, there are as yet unexplored potential mechanistic links between an imbalance of the angiotensin II receptor and angiotensin II converting enzyme (ACE2) and severe COVID-19 infections, which may also apply in SDB [11, 12].

In conclusion, our findings suggest that the sleep medicine community needs to collaborate in developing strategies for care of patients with both suspected and established SDB during major events such as the COVID-19 pandemic. Activities need to focus on the recognition of severe cases of SDB and how to initiate treatment in already identified severe cases. The potential of new technologies that enable remote monitoring to optimise treatment may be more frequently applied during times of restricted healthcare resources, in order to obtain better management of severely affected COVID-19 patients.

Ludger Grote $\oplus^{1,2}$, Walter T. McNicholas $\oplus^{3}$ and Jan Hedner ${ }^{1,2}$ on behalf of the ESADA collaborators ${ }^{4}$

${ }^{1}$ Centre for Sleep and Wake Disorders, Institute for Internal Medicine, Sahlgrenska Academy, University of Gothenburg, Gothenburg, Sweden. ${ }^{2}$ Sleep Disorders Centre, Dept of Respiratory Medicine, Sahlgrenska University Hospital, Gothenburg, Sweden. ${ }^{3}$ Dept of Respiratory and Sleep Medicine, St Vincent's Hospital Group, School of Medicine, University College, Dublin, Ireland. ${ }^{4} \mathrm{~A}$ list of the ESADA collaborators can be found in the acknowledgements section.

Correspondence: Ludger Grote, Sleep Disorders Centre, Dept of Respiratory Medicine, Sahlgrenska University Hospital, 41345 Gothenburg, Sweden. E-mail: ludger.grote@lungall.gu.se

Received: 17 April 2020 | Accepted after revision: 23 April 2020 
The ESADA collaborators are as follows ( ${ }^{*}$ : centre provided data for the study). Ulla Anttalainen and Tarja Saaresranta (Turku University Hospital, Division of Medicine, Dept of Pulmonary Diseases and Sleep Research Centre, Dept of Pulmonary Diseases and Clinical Allergology, University of Turku, Turku, Finland)"; Ozen K. Basoglu and Sezai Tasbakan (Dept of Chest Diseases, Ege University School of Medicine, Izmir, Turkey)"; Izolde Bouloukaki and Sophia E. Schiza (Sleep Disorders Centre, University of Crete, Heraklion, Greece) ${ }^{\#}$; Maria R. Bonsignore and Oreste Marrone (DiBiMIS, University of Palermo and CNR, Istituto per la Ricerca e l'Innovazione Biomedica, Palermo, Italy)"; Michel Petitjean and Gabriel Roisman (Service d'Explorations Fonctionnelles Multidisciplinaires, and Unité de Médecine du Sommeil, Hôpital Antoine Béclère, Clamart, France); Ingo Fietze, Naima Laharnar and Thomas Penzel (Centre of Sleep Medicine, Charité Universitätsmedizin Berlin, Berlin, Germany); ;an Hedner, Ding Zou and Ludger Grote (Sleep Medicine, Sahlgrenska University Hospital and Sahlgrenska Academy, Gothenburg, Sweden)"; Brian D. Kent, Walter T. McNicholas and Silke Ryan (School of Medicine and Medical Science, University College Dublin, and Dept of Respiratory and Sleep Medicine, St Vincent's Hospital Group, Dublin, Ireland); ;ohn A. Kvamme (ENT Dept, Førde Central Hospital, Førde, Norway); Sebastien Bailly, Jean-Louis Pépin and Renaud Tamisier (Université Joseph Fourier and Université Grenoble Alpes, Grenoble, France) ${ }^{\#}$; Carolina Lombardi and Gianfranco Parati (Sleep Disorders Centre, Dept of Cardiovascular Neural and Metabolic Sciences, IRCCS Istituto Auxologico Italiano, Milano-Bicocca University, Milan, Italy); Athanasia Pataka (Respiratory Failure Unit, G. Papanikolau Hospital, Thessaloniki, Greece)*; Robert Plywaczewski and Pawel Sliwinski (Institute of Tuberculosis and Lung Diseases, Warsaw, Poland); Martin Pretl (Sleep Disorders Centre, Dept of Neurology, Charles University, Prague, Czech Republic); Renata Riha (Dept of Sleep Medicine, Edinburgh Royal Infirmary, Edinburgh, UK) ${ }^{\#}$; Richard Staats (Dept of Pneumology, University Hospital de Santa Maria, Lisbon, Portugal); ; Paschalis Steiropoulos (Sleep Unit, Dept of Pneumonology, Democritus University of Thrace, Alexandroupolis, Greece); ; Pavol Joppa (Dept of Respiratory Medicine, P.J. Safarik University, Kosice, and L. Pasteur University Hospital, Kosice, Slovakia) ${ }^{\#}$; Johan Verbraecken and E. Petiet (Multidisciplinary Sleep Disorders Centre, Antwerp University Hospital and University of Antwerp, Antwerp, Belgium); ; Georgia Trakada (Pulmonary Medicine, National and Kapodistrian University of Athens, Athens, Greece) $)^{\#}$; Ondrej Ludka (Dept of Cardiology, University Hospital Brno and International Clinical Research Centre, St. Ann's University Hospital, Brno, Czech Republic) and Jitka Bušková (Department of Sleep Medicine, National Institute of Mental Health, Klecany, Czech Republic)"; Hein H (Sleep Disorders Centre, Johanniter-Krankenhaus Geesthacht, Geesthacht, Germany);; Marta Drummond and Mafalda van Zeller (Pulmonology Dept Hospital São João, Medicine Faculty of Porto University, Porto, Portugal) ${ }^{*}$; Zoran Dogas and Tea Galic (Sleep Medicine Centre, Dept of Neuroscience, University of Split School of Medicine, Split, Croatia); Haralampos Gouveris (ENT Dept at Mainz University Hospital, Mainz, Germany)"; Stefan Mihaicuta (Victor Babes University Hospital, Timisoara, Romania) ${ }^{\#}$; Winfried Randerath and Simon Herkenrath (Bethanien Hospital, Solingen, Germany); ; Francesco Fanfulla (Unità Operativa di Medicina del Sonno (Sleep Medicine Unit), Istituti Clinici Scientifici Maugeri, Padua, Italy); Dries Testelmans (Sleep Disorders Centre, University Hospital Gasthuisberg, Leuven, Belgium) ${ }^{\#}$.

The following accredited sleep centres of the German Sleep Society (DGSM) participated in the study: Sleep Centre, Neurological Dept, University Hospital, Aachen (J. Schiefer); Sleep Centre, Krankenhaus Bad Arolsen, Arolsen (W. Knüppel); Competence Centre for Sleep Medicine, Charité Universitätsmedizin Berlin, Campus Benjamin Franklin, Berlin (H. Danker-Hopfe); Florence-Nightingale Krankenhaus, Kaiserswerther Diakonie, Düsseldorf (M. Minuth); Sleep Centre, Pulmonary Medicine, Kliniken Essen Mitte, Essen (G. Nilius); Zentrum für Schlafmedizin Frankfurt, Frankfurt (H. Schneider); Klinikum Garmisch-Partenkirchen, Garmisch (R. Püschel); Krankenhaus Martha-Maria Halle, Halle (S. Schädlich); Sleep Centre, Medizinischen Hochschule, Hannover (K. Meyer); Thoraxklinik Heidelberg, University Hospital, Heidelberg (A. Benz); Sleep Centre, Pediatric Hospital St Marien, Landshut (B. Schneider); Sleep Centre, Suedharz Klinikum, Nordhausen (J. Büntzel); Sleep Centre at Sana Klinik Oldenburg, Oldenburg (I. Koper); Sleep Centre, Kreisklinik Trostberg, Trostberg (R. Koch); Sleep Centre, ENT Dept, University Hospital, Ulm (J. Lindemann).

All data are available for data sharing after de-identification of the study centre. Requests for data sharing can be sent to the first author. The original questionnaire, the analysis plan and the study protocol are available. The request for data can be forwarded after publication of the research letter.

Support statement: This work was supported by a European Respiratory Society CRC grant and by Bayer through a collaboration agreement with the ESADA network. Funding information for this article has been deposited with the Crossref Funder Registry.

Conflict of interest: L. Grote reports a collaboration between the ESADA and Bayer in part related to the submitted work, has received non-financial support for research from and has been on speakers' bureau for Itamar Medical, Resmed, Philips and AstraZeneca outside the submitted work; and has a patent on sleep apnoea therapy issued and licensed. W.T. McNicholas has nothing to disclose. J. Hedner reports grants from ResMed, Respironics, European Respiratory Society and Bayer, during the conduct of the study; personal fees for lectures from Philips Respironics, Bayer and MSD, outside the submitted work; and has a patent for Pharmacological therapy in OSA licensed.

\section{References}

1 Benjafield AV, Ayas NT, Eastwood PR, et al. Estimation of the global prevalence and burden of obstructive sleep apnoea: a literature-based analysis. Lancet Respir Med 2019; 7: 687-698.

2 Hedner J, Grote L, Bonsignore M, et al. The European Sleep Apnoea Database (ESADA): report from 22 European sleep laboratories. Eur Respir J 2011; 38: 635-642.

3 Chen $\mathrm{T}, \mathrm{Wu} \mathrm{D}$, Chen $\mathrm{H}$, et al. Clinical characteristics of 113 deceased patients with coronavirus disease 2019: retrospective study. BMJ 2020; 368: m1091.

4 Baker JG, Sovani M. Case for continuing community NIV and CPAP during the COVID-19 epidemic. Thorax 2020; 75: 368.

5 Swedish Perioperative Registry (SPOR). Publication of actual statistics on elective surgical procedures performed in Sweden on a weekly basis before and during the COVID-19 pandemic (for weeks 1-18 2020, entire country and 
different healthcare regions). Report from the Swedish Broadcast SVT. Available from: www.svt.se/datajournalistik/ corona-uteblivna-operationer/ Date last accessed: 11 May 2020.

6 British Thoracic Society. Advice for those seeing patients with obstructive sleep apnoea. Available from: www. brit-thoracic.org.uk/about-us/covid-19-information-for-the-respiratory-community/ Date last updated: 23 March 2020.

7 American Academy Sleep Medicine. COVID-19 mitigation strategies for sleep clinics and labs. https://aasm.org/ covid-19-resources/ Date last updated: 8 April 2020.

8 Gonzalez J, Maisonobe J, Oranger $\mathrm{M}$, et al. Les procedures du $\mathrm{GAVO}_{2}$ (Groupe assistance ventilatoire et oxygène). Appareillages respiratoires de domicile d'un malade suspect ou porteur du de virus respiratoire COVID-19 $\left[\mathrm{GAVO}_{2}\right.$ procedures (French scientific group on chronic ventilation and oxygen). Homecare respiratory equipment for patients suspected to be infected with the respiratory virus COVID-19]. Available from: http://splf.fr/gavo2/ English version updated February 2020. French version updated April 2020.

9 Grote L, Albrecht D, Franklin K, et al. Vägledning angående coronavirus (COVID-19) och obstruktiv sömnapné (OSA) [Guidance on coronavirus (COVID-19) and obstructive sleep apnea (OSA)]. Expert group statement. Available from: www.sesar.se Date last updated: March 2020.

10 Simonds AK, Hanak A, Chatwin $\mathrm{M}$, et al. Evaluation of droplet dispersion during non-invasive ventilation, oxygen therapy, nebuliser treatment and chest physiotherapy in clinical practice: implications for management of pandemic influenza and other airborne infections. Health Technol Assess 2010; 14: 131-172.

11 Liu PP, Blet A, Smyth D, et al. The science underlying COVID-19: implications for the cardiovascular system. Circulation 2020; in press [https://doi.org/10.1161/CIRCULATIONAHA.120.047549].

12 Weiss JW, Tamisier R, Liu Y. Sympathoexcitation and arterial hypertension associated with obstructive sleep apnea and cyclic intermittent hypoxia. J Appl Physiol 2015; 119: 1449-1454. 